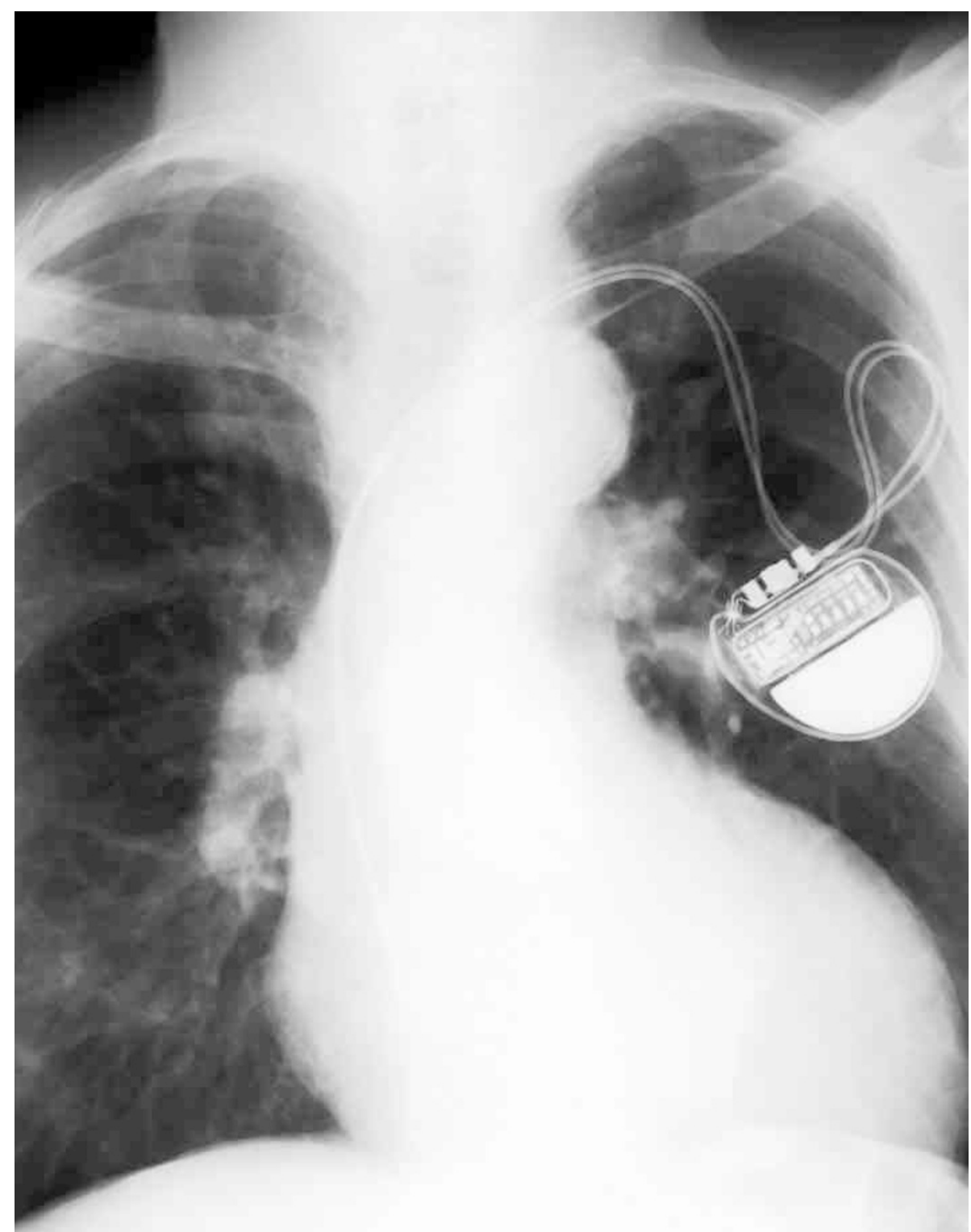

Artificial pacemakers regulate irregular heart beats, which cause up to $20 \%$ of embolic strokes.

PHYSIOLOGY

\title{
Beating stroke
}

\section{New drugs and more focused therapy might cut down on atrial fibrillation and reduce the incidence of stroke.}

\section{BY NEIL SAVAGE}

$\mathrm{E}$ ncounters that set the heart aflutter are celebrated in love songs and romance literature. But that fluttery feeling can also be the first sign of a dangerous episode of atrial fibrillation (AF), the most common form of irregular heartbeat. And while the symptoms might be mild, or even unnoticeable, AF is a leading cause of heart disease and stroke.

The incidence of AF is high and rising, in

part because it's age-related and the population is ageing. One in four people in the United States over the age of 40 will experience AF, as will many Europeans ${ }^{1,2}$. The American Heart Association (AHA) estimates that 2.7 million

\section{DNATURE.COM}

For the latest research on atrial fibrillation:

go.nature.com/vn3rgx people in the United States had AF in 2010, and this number will rise to 12 million by 2050 .

Fortunately, doctors are learning more about the condition and finding better ways to treat it, from surgery to new drugs. They're also learning that more people might be at risk of or might actually have, the disease than previously thought.

The atria are the upper two chambers of the heart. Blood that is depleted of oxygen enters the right atrium and is pumped into the right ventricle - the lower chamber which in turn pumps it to the lungs to pick up a fresh supply of oxygen. The oxygenated blood returns to the heart and enters the left atrium, passing through the left ventricle to the aorta for distribution around the body. The whole system is orchestrated by electrical signals to the heart muscle. In one form of AF, two or three heart cells start firing off 300-400 electrical impulses per minute; the impulses reach neighbouring cells, sending a wave of erratic electrical signals through the heart muscle. The atrial beating becomes rapid and chaotic, and out of sync with the ventricles. In other cases, an underlying disorder - such as diabetes, hypertension or sleep apnoea — damages the heart muscle, disrupting electrical signals. But in both forms the ultimate effect is similar: atrial fibrillation is "a very chaotic, disordered activity in the human heart”, says Sanjiv Narayan, a cardiologist at the University of California, San Diego.

Atrial fibrillation can be fleeting or permanent; it can last from minutes to hours and stop on its own, or recur regularly, or the heart's rhythm can always be abnormal. The effect can be so mild that it goes unnoticed, but it can also cause palpitations, shortness of breath and weakness, even brief loss of consciousness. Worse, the rapid, irregular heartbeat can lead to heart failure. It also often causes blood clots to form inside the heart. Such clots can travel into the arteries and block the blood supply to the brain, resulting in an embolic stroke; AF causes 15-20\% of this form of stroke, and increases the risk of stroke overall by a factor of five, according to the AHA.

\section{BALANCING THE BEATS}

To control the heartbeat, doctors often prescribe anti-arrhythmia drugs, or in some cases perform open-heart surgery to make small cuts in the heart tissue where the erratic electrical signals originate. Since the mid1990s, doctors have also performed a procedure called catheter ablation. They thread a thin tube from the groin to the heart and deliver energy (usually as radiofrequency waves or ultrasound) that warms the tissue. The directed energy is generally aimed at areas around the pulmonary veins, which carry blood from the lungs to the heart and where many of the fibrillation triggers are thought to originate. Both procedures form scar tissue that isn't as conductive as the original heart muscle, isolating the electrical triggers from the rest of the heart and blocking the signal. 
Small studies of catheter ablation show it to be effective in treating a large proportion of AF patients. But the larger picture is still unclear. There has never been a large, multicentre trial to assess cases of AF complicated by underlying disease, says Douglas Packer, a specialist in cardiovascular disease at the Mayo Clinic in Rochester, Minnesota. Packer hopes to fill that gap: he is the lead investigator of CABANA (Catheter Ablation Versus Antiarrhythmic Drug Therapy for Atrial Fibrillation), a study that plans to compare catheter ablation and drug treatment in 3,000 patients over a period of 2-5 years ${ }^{3}$.

In a pilot study — presented at the 2010 American College of Cardiology Scientific Sessions - that followed 60 patients for a year, $65 \%$ of those who underwent ablation had no recurrence of symptoms, compared with only $41 \%$ of those taking anti-arrhythmia drugs. CABANA will examine how ablation compares with drugs at reducing AF-related death, dementia, which some studies have linked to AF, and hospital stays. "The big deal about CABANA," Packer says, "is it looks at mortality, it looks at stroke, it looks at hospitalization costs, it looks at quality of life, and not just at recurrence of atrial fibrillation."

\section{NOISE REDUCTION}

Ablation technology is still being improved. Narayan, for example, has pioneered a more precise version of the procedure ${ }^{4}$. A computer algorithm studies electrocardiograms that record the heart's electrical patterns, and sorts out the sources of the disruption, instead of assuming it's coming from the pulmonary veins. Narayan discovered small areas of electrical waves called rotors, which set off the disruption. Using his algorithm to map rotors in a patient's heart, Narayan burns the tissue at the sources of the arrhythmia. "If you find them, you can shut down atrial fibrillation pretty fast," Narayan says. In several cases, he says, he has been able to stop AF within one to three minutes of onset.

Narayan compared two sets of patients, one that underwent his technique followed immediately by conventional ablation, and one that had conventional ablation alone. After two years, $84 \%$ of those who had both procedures were symptom-free, compared with only half of those who only had conventional ablation ${ }^{5}$.

Although stopping AF can improve patients' lives, it's still not clear whether it extends life. "Precious few therapies for AF have reduced stroke risk, which is a tragedy and a bit of a mystery," says Narayan.

The AF connection to stroke is gaining more attention. "In many ways, the biggest publichealth issue with AF is the stroke side of it," says Christopher Granger, a cardiologist at Duke University in Durham, North Carolina. "The burden of atrial fibrillation is increasing, and there's a recognition that there's a major gap in efforts to prevent stroke."

\section{A FEARFUL FLUTTER}

These electrocardiograms show the regular atrial impulse in a normal heart rhythm (left) and the erratic pulses in atrial fibrillation.

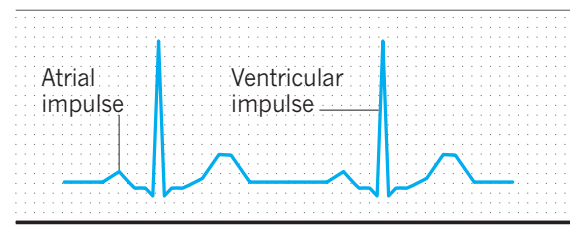

Normal heart rhythm

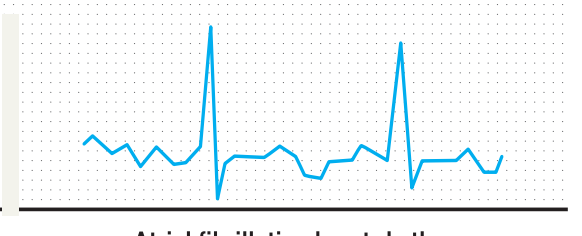

Atrial fibrillation heart rhythm
To reduce the risk of stroke, doctors often prescribe the blood-thinning drug warfarin to prevent clots from forming. Warfarin has significant drawbacks, however. It attacks vitamin $\mathrm{K}$, which creates blood factors that promote coagulation. But diets high in vitamin $\mathrm{K}$, found in green vegetables such as broccoli, can interfere with warfarin. And the drug can cause bleeding in the brain. Patients on warfarin, therefore, need regular blood tests to ensure a precise concentration of the drug in blood. "It's very effective, but it's very much of a challenge," says Granger. "Patients don't like it, and doctors don't like it." As a result, roughly half the people who could be benefiting from warfarin as an AF treatment are not taking it.

New drugs interfere with blood clotting

"Precious few

therapies for

atrial fibrillation

have reduced

stroke risk,

which is a

tragedy and a

bit of $a$

mystery."

factors at a later stage in the coagulation cascade. In the past two years, two drugs - dabigatran and rivaroxaban - have been approved for treating AF patients in the United States and Europe. A third, apixaban, received a recommendation for approval for AF patients in Europe in September 2012, and approval by the US Food and Drug Administration (FDA) is expected. "I'm confident that the FDA will be approving the drug," says Granger, who led a study that found apixaban did better than warfarin at preventing stroke and caused less bleeding. Another anticoagulant, edoxaban, is still in phase III clinical trials.

"They are exciting because that does offer progress for patients," Kathryn Wood, a professor of nursing at Duke, says of the new drugs. "We're still not clear on whether they're for everybody."

Wood was part of an expert panel that issued guidelines for the AHA and the American Stroke Association ${ }^{7}$. The guidelines say that dabigatran, rivaroxaban and apixaban should be considered for patients with AF. Wood says that there's some hesitancy among healthcare providers because there's no test to see how much of the drug is in the blood stream, and because there's no known way to quickly reverse their anticlotting effects if a patient is injured or needs surgery. It might be best for patients doing well on warfarin to stay on that conventional medication, she says.

\section{EXPANDING TREATMENT}

The adoption of safer, better drugs could expand the use of anti-coagulation therapy. That might be useful, as AF's prevalence now seems to be higher than had previously been thought, says cardiologist Jeff Healey of McMaster University in Hamilton, Ontario. Healey gathered data from pacemakers in 2,580 patients with high blood pressure, who had never been diagnosed with AF. He detected episodes of fibrillation that lasted at least 6 minutes in 261 of them ${ }^{8}$. "Either patients have no symptoms and don't know to complain, or they're transient and by the time they get an electrocardiogram, it's gone," Healey says.

This sort of subclinical AF might cause some of the $25 \%$ of embolic strokes in which the cause isn't known. Of course, Healey says, the people in his study already have pacemakers, so they have known heart problems. He's expanding his study to include people at risk for $\mathrm{AF}$ but without pacemakers. If doctors can identify more people with subclinical AF, and thus at risk for stroke, anti-coagulant therapy might help them, Healey says.

"You don't have to prevent too many strokes to make it worthwhile to pay for such things," he says. "One stroke is the difference between living at home with your spouse and institutional care, which is terribly expensive."

Neil Savage is a science writer based in

Lowell, Massachusetts.

1. Lloyd-Jones, D. M. et al. Circulation 110, 10421046 (2004).

2. Heeringa, J. et al. Eur. Heart J. 27, 949-953 (2006).

3. Rettmann, M. E. et al. Acad. Radiol. 19(11), 1332-1344 (2012)

4. Narayan, S. M. et al. J. Am. Coll. Cardiol. 60, 628-636 (2012).

5. Narayan, S. M. et al. EP Lab. Digest 12(2), 1-10 (2012)

6. Granger, C. B. et al. N. Engl. J. Med. 365, 981-992 (2011).

7. Furie, K. L. et al. Stroke 12, 3442-3453 (2012); published online 2 August 2012.

8. Healey, J. S. et al. N. Engl. J. Med. 366, 120-129 (2012). 